Check for updates

Cite this: RSC Adv., 2019, 9, 35976

Received 26th July 2019

Accepted 21st October 2019

DOI: 10.1039/c9ra05813c

rsc.li/rsc-advances

\section{Near-infrared-induced IR780-loaded PLGA nanoparticles for photothermal therapy to treat breast cancer metastasis in bones}

\author{
Jingyi Li, D $\dagger^{\mathrm{a}}$ Huiyu Hu, $\hat{\dagger}^{\mathrm{b}}$ Zichao Jiang, ${ }^{\mathrm{a}}$ Sijie Chen, ${ }^{\mathrm{c}}$ Yixiao Pan, ${ }^{\mathrm{a}}$ Qi Guo, ${ }^{a}$ Qiqi Xing, ${ }^{\mathrm{a}}$ \\ Zhaocheng Jing, ${ }^{a}$ Yihe $\mathrm{Hu}^{* a}$ and Long Wang (D) *a
}

\begin{abstract}
Nanodrug-based cancer therapy, especially when treating bone metastases, faces the problem of limited therapeutic efficacy. In this work, we reported a photothermally triggered nanomaterial based on IR780entrapped poly-lactide-co-glycolide (PLGA) nanoparticles (IR780@PLGA NPs) for the photothermal therapy of bone metastases of breast cancer, in which IR780 converted light into heat to play a role in "burning" the tumors. Anti-tumor therapy studies showed the impressive effectiveness of IR780@PLGA NPs in the photothermal therapy (PTT) of bone metastases. As a result, the IR780@PLGA NPs show a great potential for controlling the bone metastases of breast cancer
\end{abstract}

\section{Introduction}

Breast cancer, which has a high probability of bone metastasis, is a malignant disease with the highest incidence among women worldwide; it is the leading cause of cancer death among women worldwide and accounts for an estimated $11.6 \%$ of the total number of cancers diagnosed in 2018. ${ }^{1}$ Bone metastasis, which occurs in the middle and late stages of breast cancer, ${ }^{2,3}$ is reported in more than $70 \%$ of patients with breast cancer $^{4}$ and is responsible for substantial skeletal disease morbidity, ${ }^{5,6}$ including bone loss, ${ }^{5,6}$ hypercalcaemia, neurological compression, pathological fracture, and severe pain, leading to mortality in many individuals. ${ }^{2,7-9}$ Therapies exist for breast cancer metastasis to bone, such as surgery and chemotherapy; however, these therapies have distinct disadvantages. ${ }^{\mathbf{1 0 - 1 2}}$ Surgery is only suitable for removing primary lesion tumours that are well-defined; it is not an ideal approach for the treatment of small and poorly defined metastases. ${ }^{11}$ Also, the problem of the poor weight-bearing of the remaining bones still remains. ${ }^{13}$ Chemotherapy, which is the most widely applied therapy for bone metastases, possesses the advantages of less injury than surgery and greater suitability for the middle and late stages of breast cancer; however, it is still substantially limited by high visceral toxicity and drug resistance, ${ }^{\mathbf{1 1}}$ and the

\footnotetext{
${ }^{a}$ Department of Orthopedics, Xiangya Hospital, Central South University, Changsha, Hunan 410008, China.E-mail: huyh1964@163.com; dr_wanglong@csu.edu.cn; Tel: +86 13973184558; +86 13974931684

${ }^{b}$ Department of General Surgery, Xiangya Hospital, Central South University, Changsha, Hunan 410008, China

${ }^{c}$ Departmen of Ultrasound Diagnosis, The Second Xiangya Hospital, Central South University, Changsha, Hunan 410011, China

$\dagger$ These authors contributed equally.
}

high risk of cancer relapse also remains. ${ }^{14}$ Moreover, the bone marrow has a rich microenvironment that serves as a reservoir for dormant tumour cells, ${ }^{3,15}$ facilitates the survival of cancer cells and mediates drug resistance. ${ }^{\mathbf{2 , 6 , 1 6 , 1 7}}$ For patients with bone metastases, traditional therapy is associated with substantial side effects and unsatisfactory treatment effects. ${ }^{12,15}$ As a result, it is necessary to develop a way to inhibit breast tumour growth and the progression of bone metastases.

Nanomedicines, which have been used in therapy, including gene therapy, radiotherapy, chemotherapy and photothermal therapy (PTT), are a considerable innovation for cancer therapy. ${ }^{\mathbf{1 1} 18}$ Recently, due to its minimized invasiveness, improved preservation of the surrounding tissues and high anti-tumour efficiency compared with traditional treatments, ${ }^{19,20}$ near-infrared (NIR) laser-induced PTT has attracted increasing attention as an emerging strategy for the local treatment of tumours by generating high temperatures in tissues, thus leading to more precise damage to the targeted regions. ${ }^{5,18,21}$ NIR laser-induced PTT uses photo-absorbance to transform light energy into heat to "burn" cancer cells. ${ }^{11,22}$ Several nanomaterials have been developed as PTT agents due to their excellent NIR light-absorption properties. ${ }^{\mathbf{1 1 , 1 2 , 1 9}}$ However, some of these nanomaterials are potentially toxic due to poor biodegradability and biocompatibility, inevitably limiting their clinical applications. ${ }^{19,23}$ Thus, it is important to develop a biodegradable photoabsorbent for biologically safe NIR laser-induced PTT. More importantly, although PTT has been widely used to treat superficial tumours, reports of its use as a treatment for deep tumours are still uncommon, necessitating the investigation of the effects of PTT for the treatment of deep tumours, such as the bone metastases of breast cancer.

IR780 iodide, which converts NIR (750-1000 nm) laser energy into heat after irradiation, is a lipophilic heptamethine 
NIR absorber with a characteristic absorption peak at $780 \mathrm{~nm} \cdot{ }^{24-27}$ It was found to have extraordinary intrinsic tumor targeting properties without further modification. ${ }^{28}$ However, IR780 iodide has several limitations, including poor aqueous stability and photostability, which may severely impede its further clinical applications. ${ }^{24,29,30}$ Additionally, the toxicity of IR780 cannot be ignored because of its maximum tolerable dose of $1.5 \mathrm{mg} \mathrm{kg}^{-1}$ in mice. ${ }^{26,31}$ To overcome these limitations, some investigators have encapsulated IR780 iodide in various nanoparticles (NPs). ${ }^{23,25,29,32}$ This approach capitalizes on the ability of NPs to protect the loaded IR780..$^{23,25,29,32,36,37}$ Moreover, some investigators have demonstrated that IR780-loaded NPs effectively suppress the metastasis of breast tumours to lungs under an NIR laser. ${ }^{5,734}$ IR780 iodide encapsulated in poly(lactic-coglycolic acid) (PLGA) NPs as a PTT agent for cancer therapy has interested investigators because of its outstanding performance in photothermal conversion. PLGA has been reported to coencapsulate a variety of hydrophobic and hydrophilic materials, which enhances its therapeutic anti-tumour efficacy due to its core-shell construction. ${ }^{11,22,35}$ Some researchers have used PLGA NPs for PTT therapy in treating superficial tumors and have reported satisfactory results. ${ }^{11,22,33,35}$ However, the efficiency of IR780 iodide encapsulated in PLGA NPs for PTT therapy of bone metastases induced by breast cancer requires further study. In this study, we encapsulated IR780 into PLGA NPs to improve the biocompatibility and stability of PTT. Furthermore, we performed intra-tumoural injections to avoid systemic toxicity. Therefore, the accumulation of heat at the tumour site was achieved upon irradiation without injuring healthy tissues.

In this study, we developed a photothermally triggered IR780 nanotheranostic system based on IR780-encapsulated PLGA NPs (IR780@PLGA NPs) to treat bone metastases induced by breast cancer. In the NPs, IR780 could absorb and convert NIR light energy into heat. ${ }^{11}$ In particular, the IR780@PLGA NPs could be exploited as an adjuvant therapy to treat cancer with bone metastases. Because of their outstanding photothermal conversion properties, the IR780@PLGA NPs exhibited excellent PTT for bone metastases. Therefore, we hypothesized that PTT via IR780@PLGA NPs combined with NIR irradiation is a promising alternative therapy that is suitable for the treatment of bone metastases induced by breast cancer. This study was designed to explore the effectiveness of PTT using IR780@PLGA NPs in an intraosseous model of breast cancer metastasis to bone.

\section{Experimental}

\section{Materials, cells and animals}

IR-780 iodide, PLGA (lactide $:$ glycolide $=50: 50, M_{\mathrm{w}}=10000$ ), and polyvinyl alcohol (PVA, $M_{\mathrm{w}}=30000-70000$ ) were all obtained from Sigma-Aldrich (USA). Other reagents were of analytical grade and were used without further purification. 4T1 mouse breast cancer cells and human bladder epithelial cells (SV-HUC-1) were obtained from Xiangya Hospital at Central South University (China) and cultured according to recommendations.
All experiments involving the use of animals were performed in accordance with the guidelines of the Department of Laboratory Animals, Central South University, China, and approved by the Ethics Committee of Central South University in China. To obtain mice with bone metastases of $4 \mathrm{~T} 1$ tumours, approximately $1 \times 10^{5} 4 \mathrm{~T} 1$ cells (murine breast cancer cells) were inoculated into the medullary cavity of the right tibia of female BALB/c mice ( 6 weeks old with an average body weight of $20 \mathrm{~g}$ ). The widths and lengths of the tumours were measured, and a formula was used to calculate the tumour volumes as follows: tumour volume $=0.5 \times$ width $^{2} \times$ length.

\section{Preparation of IR780@PLGA NPs}

First, $3 \mathrm{~mL}$ of dichloromethane and $100 \mathrm{mg}$ of PLGA were mixed and thoroughly stirred. After $2 \mathrm{mg}$ of IR7 80 and $15 \mathrm{~mL}$ of $4 \% \mathrm{w} / \mathrm{v}$ cold PVA solution were sequentially added, the solution was emulsified with an ultrasonic processor for $2 \mathrm{~min}$. The resulting emulsion was mixed in $20 \mathrm{~mL}$ of deionized water and stirred in order to fully volatilize the dichloromethane. Finally, after washing three times with deionized water (10 $000 \mathrm{rpm}, 7 \mathrm{~min}$ ), the resulting NPs were stored at $4{ }^{\circ} \mathrm{C}$. All procedures were performed in the dark in an ice bath (Fig. 1).

\section{Characterization}

The IR780@PLGA NPs were irradiated with an 808 nm NIR laser (T808F2W, Minghui Optoelectronic Technology, China). Before laser irradiation, the morphologies and structures of the NPs were observed by a scanning electron microscope (SEM, JEOL-7800F) and a transmission electron microscope (TEM, Hitachi H-7600). The size distribution of the NPs was measured by a Nano ZS dynamic light scattering analyser (Malvern Instruments, UK). The UV-vis-NIR absorption spectra of the PLGA NPs, free IR780 and IR780@PLGA NPs (0.2 $\mathrm{mL}$ of each sample in 96-well plates, equivalent IR780 concentration of $60 \mu \mathrm{g} \mathrm{mL}^{-1}$ ) were obtained at room temperature. A steady-state spectrophotometer (QuantaMasterTM 40, USA) was used to ensure high absorption in the NIR region and to monitor the IR780 content. The encapsulation

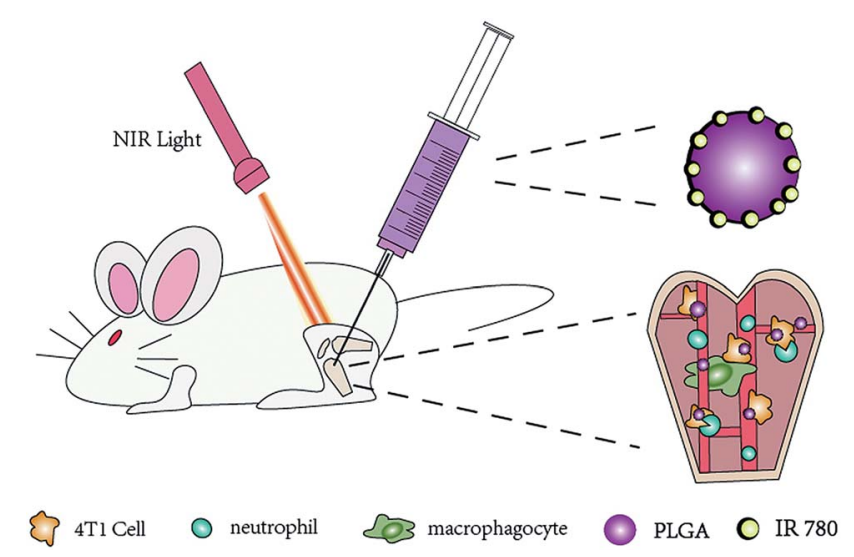

Fig. 1 A schematic of PTT using IR780@PLGA NPs with NIR lasercontrolled IR780 release. 
efficiency of IR780 in the IR780@PLGA NPs was calculated as the weight ratio of encapsulated IR780 to the total added amount of IR780. The amount of encapsulated IR780 was calculated as the difference between the total added amount of IR780 and the amount recovered in the washed supernatant during preparation.

The IR780 loading and entrapment efficiency of the IR780@PLGA NPs were calculated using the following equations:

$$
\begin{aligned}
& \text { IR780 loading }(\%) \\
& =\text { Amount of IR780 encapsulated in NPs/Mass of NPs } \times 100 \%
\end{aligned}
$$

and

$$
\begin{aligned}
& \text { Encapsulation efficiency }(\%) \\
& =\text { Amount of IR780 encapsulated in NPs/Total added } \\
& \text { amount of IR780 } \times 100 \%
\end{aligned}
$$

\section{In vitro PTT effects}

The temperature profile of the IR780@PLGA NPs was monitored using an infrared thermal imaging camera (FLIR C2, USA) under laser irradiation. IR780@PLGA NPs, free IR780, blank PLGA NPs, and phosphate-buffered saline (PBS, serving as the negative control) were placed in a $1.5 \mathrm{~mL}$ Eppendorf (EP) tube ( $1 \mathrm{~mL}$ of each sample) and exposed to an $808 \mathrm{~nm}$ NIR laser at $2.0 \mathrm{~W} \mathrm{~cm}^{-2}$ for $10 \mathrm{~min}$ (T808F2W, Xi'an Minghui Optoelectronic Technology, China). The amount of IR780 was equivalent in the free IR780 and IR780@PLGA NP samples (60 $\mu \mathrm{g} \mathrm{mL}^{-1}$ IR780), and the amount of PLGA was equivalent in the blank PLGA NP and IR780@PLGA NP samples (9 $\mathrm{mg} \mathrm{mL}^{-1}$ PLGA). The temperature of the solutions was recorded every 30 seconds. Moreover, the effects on the photothermal efficiency of the IR780@PLGA NPs were also investigated by studying different IR780 concentrations $\left(6,12,30\right.$ and $\left.60 \mu \mathrm{g} \mathrm{mL}{ }^{-1}\right)$ and laser power densities $\left(1.0,2.0\right.$ and $\left.3.0 \mathrm{~W} \mathrm{~cm}^{-2}\right)$.

\section{Cell experiments}

The biocompatibility of the IR780@PLGA NPs and their photothermal ablation of tumor cells in vitro were evaluated by the Cell Counting Kit (CCK-8) assay. Incubation of 4T1 cells at a density of $1 \times 10^{4}$ cells per well in 96-well plates was performed with different IR780 concentrations. Then, the cells were incubated for $6,12,24$, and $48 \mathrm{~h}$ after irradiation with an NIR laser ( $\left.808 \mathrm{~nm}, 2.0 \mathrm{~W} \mathrm{~cm}^{-2}, 10 \mathrm{~min}\right)$. The cells were washed twice with PBS. Then, the cell viability was evaluated using a cell counting kit-8 (CCK-8) assay. The cell viability of SVHUC-1 incubated with IR780@PLGA NPs for $24 \mathrm{~h}$ with or without NIR irradiation was set as a control. The cell viability was defined as the percentage of surviving cells versus untreated cells. In addition, $4 \mathrm{~T} 1$ cell viability under different intensities of laser irradiation was evaluated. SV-HUC-1 and $4 \mathrm{~T} 1$ cell viabilities with different concentrations of doxorubicin $(\mathrm{DOX})^{5,38}$ were also evaluated in order to compare the cytotoxicities of the chemotherapy drug and IR780@PLGA NPs.
Confocal laser scanning microscopy (CLSM, Zeiss LSM 510) was employed to estimate the cell apoptosis after NIR irradiation. 4T1 cells were cultured on a confocal imaging dish at a density of $1 \times 10^{6}$ cells per ml. After $24 \mathrm{~h}$ of incubation, the cells were treated with $200 \mu \mathrm{l}$ IR780@PLGA NPs for $3 \mathrm{~h}$ in an incubator. Then, the cells were incubated with DAPI and imaged to measure the photothermal ablation of the tumor cells. The $4 \mathrm{~T} 1$ cells were incubated in confocal imaging dishes for $24 \mathrm{~h}$, similar to the preceding steps, and the dishes were divided into 8 groups according to the different IR780 concentrations $\left(0.06,0.3,0.6\right.$ and $1.5 \mu \mathrm{g} \mathrm{mL} \mathrm{m}^{-1}$ ), with or without NIR irradiation $(n=3)$. The NIR groups were irradiated with the $808 \mathrm{~nm}$ NIR laser at $2 \mathrm{~W} \mathrm{~cm}^{-2}$ for $10 \mathrm{~min}$. Finally, the cells were stained with DAPI and the Calcein AM-PI double staining kit and imaged to evaluate the cell killing efficiency.

\section{In vivo anti-tumour therapy}

Before therapy was performed, the tumour-bearing legs of the mice were observed using a Siemens S3000 US scanner. All legs, including tumour-bearing legs and normal legs, were imaged using B-mode US and CEUS-mode US. When damage of the bone caused by tumors was first observed, as indicated by the ultrasound, the mice were randomly divided into the following five groups ( $n=5)$ : (1) PBS, (2) blank PLGA NPs, (3) IR780@PLGA NPs, (4) blank PLGA NPs with laser irradiation, and (5) IR780@PLGA NPs with laser irradiation. Saline (0.2 mL) containing PLGA NPs or IR780@PLGA NPs was intra-tumourally injected into the mice. All NPs containing IR780 were at an IR780 concentration of $60 \mu \mathrm{g} \mathrm{mL}^{-1}\left(0.6 \mathrm{mg} \mathrm{kg}^{-1}\right.$ per mouse). At $5 \mathrm{~min}$ after injection, the tumours of the mice in groups 1-5 were irradiated with the $808 \mathrm{~nm}$ laser $\left(2.0 \mathrm{~W} \mathrm{~cm}^{-2}, 5 \mathrm{~min}\right)$. During irradiation, temperature changes in the tumour tissue were monitored by an infrared thermal imaging camera (FLIR C2, USA). The body weights and tumour volumes were measured before injection every other day for 8 days. One day later, the mice in each group were sacrificed by cervical dislocation. Important organs and tumour-bearing legs were harvested, and staining of the organs was performed with haematoxylin and eosin (H\&E); meanwhile, the tumour-bearing legs were observed using micro CT and stained with H\&E.

\section{Statistical analysis}

All data are presented as the mean values \pm standard deviations from at least three separate experiments. The data were analysed by one-way ANOVA and Student's $t$-test using SPSS 17.0 and GraphPad Prism 5 software. ${ }^{*} p<0.05$ was considered to be statistically significant for the differences.

\section{Results and discussion}

\section{Characterization}

The SEM and TEM images confirmed the spherical structure of the IR780@PLGA NPs (Fig. 2A). As shown in Fig. 2A and B and Table 1, the IR780@PLGA NPs were spherical in shape and $260.6 \pm 4.8 \mathrm{~nm}$ in diameter with a narrow size distribution (PDI: $-2.35 \pm 0.02$ ); this was also observed for PLGA-based core-shell 
A
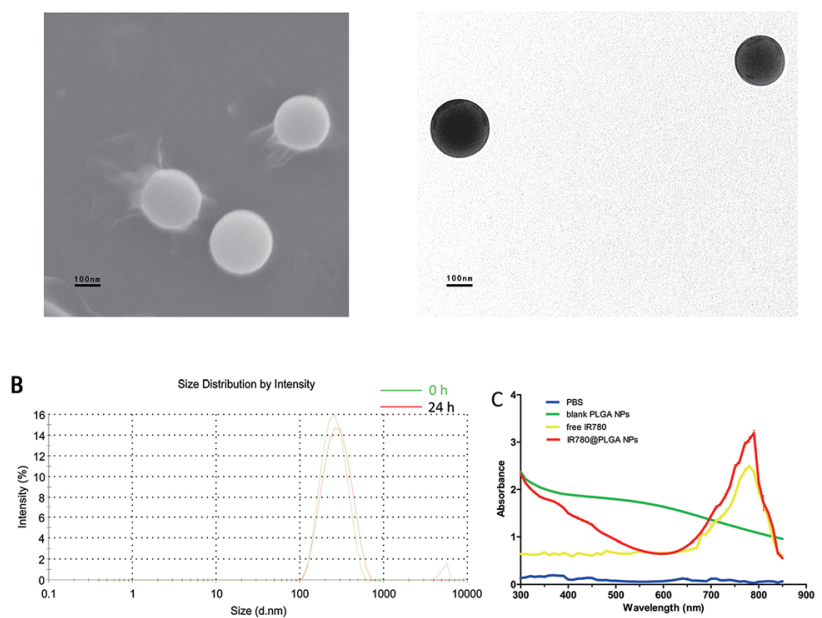

Fig. 2 (A) SEM (left) and TEM (right) images of the IR780 (aPLGA NPs (B) The size distribution of the IR780@PLGA NPs. (C) UV-vis-NIR absorption spectra of PBS, blank PLGA NPs, free IR780 and IR780@PLGA NPs.

micelles in many previous studies. ${ }^{11}$ Additionally, as shown in Table 1, the zeta potential of the NPs was $-0.17 \pm 0.06 \mathrm{mV}$.

As shown in Table 1, for the IR780@PLGA NPs at a concentration of $60 \mu \mathrm{g} \mathrm{mL} \mathrm{g}^{-1}$, the IR780 encapsulation efficiency was $31.2 \pm 1.2 \%$ and the IR780 loading content was $6.6 \pm 1.8 \mu \mathrm{g}$ $\mathrm{mg}^{-1}$. As shown in the ultraviolet-visible (UV-vis)-NIR absorption spectra of the IR780@PLGA NPs and their components (Fig. 2C), the absorption spectrum of free IR780 showed a peak at approximately 780 to $790 \mathrm{~nm}$, whereas the IR780@PLGA NPs showed an absorption peak in approximately the same area. For the blank PLGA NPs, absorption peaks were not observed from $400 \mathrm{~nm}$ to $900 \mathrm{~nm}$, suggesting that PLGA itself does not have the ability to absorb light and confirming that IR780-loaded NPs can be used as a suitable photoabsorbent.

\section{In vitro PTT effects}

To investigate the PTT effects of the IR780@PLGA NPs, IR780@PLGA NPs at different concentrations, free IR780, blank PLGA NPs, and PBS were exposed to $808 \mathrm{~nm}$ NIR laser irradiation with power densities of 1.0, 2.0 and $3.0 \mathrm{~W} \mathrm{~cm}^{-2}$ for $10 \mathrm{~min}$. As shown in Fig. 3A, no obvious change was observed in the temperature of the PBS and blank PLGA NPs after 10 min of NIR irradiation. In contrast, due to the photothermal effects of IR780, a significant temperature increase was observed under NIR irradiation. The maximum temperature achieved for the free IR780 solutions was $47.4^{\circ} \mathrm{C}$. Similarly, obvious temperature
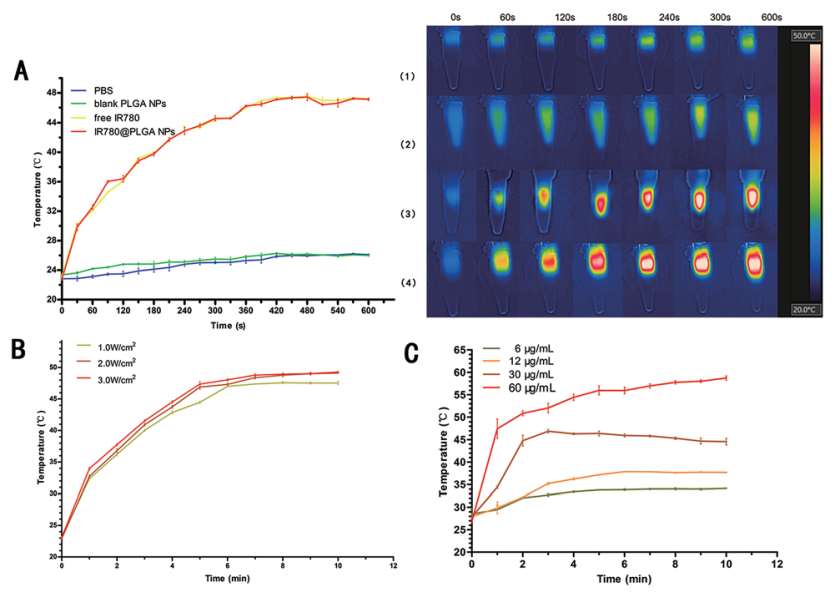

Fig. 3 Temperature variation curves. (A) Temperature variation curves (left) and infrared thermal images (right) of (1) PBS, (2) blank PLGA NPs, (3) free IR780 and (4) IR780@PLGA NPs. (B) Temperature variation curves of the IR780@PLGA NPs with varying NIR power density (1.0, 2.0 and $3.0 \mathrm{~W} \mathrm{~cm}^{-2}$ for $10 \mathrm{~min}$ ). (C) Temperature variation curves of the IR780@PLGA NPs at PBS concentrations of 6, 12, 30 and $60 \mu \mathrm{g}$ $\mathrm{mL}^{-1}$.

increases were observed for IR780@PLGA NP solutions after irradiation, and the temperature increased to $47.3{ }^{\circ} \mathrm{C}$; this allows the NPs to act as an effective photothermal nanoagent for PTT because the temperature threshold that is supposed to cause irreversible damage to cancer tissue is $43{ }^{\circ} \mathrm{C} ., 39$ The IR780@PLGA NPs showed nearly the same PTT efficiency as free IR780, indicating that PLGA encapsulation has no impact on the PTT effect of IR780. Moreover, the increase in the temperature of the IR780@PLGA NP solution could be controlled by varying the concentration of IR780 and the laser power density, as expected (Fig. 3B and C). Therefore, due to the excellent PTT performance of the IR780@PLGA NPs, they are promising PTT agents for the treatment of tumours.

\section{In vitro anti-tumour activity}

To estimate the in vitro anti-tumour therapeutic efficacy of the IR780@PLGA NPs, the viability of 4T1 cells was analysed by CCK-8 assay. As shown in Fig. 4, with NIR irradiation, the cell viability decreased with increasing concentration of NPs. Moreover, the cell viability in the groups decreased with increasing concentration of NPs, even in the absence of laser irradiation; this indicates that the IR780@PLGA NPs are cytotoxic $(p<0.05)$. After the same concentration of NPs was exposed to NIR irradiation, the cell viability was less than that without irradiation. After $4 \mathrm{~T} 1$ cells were incubated with the

Table 1 Characteristics of the blank PLGA NPs and IR780 @PLGA NPs $(n=3)$

\begin{tabular}{llllll}
\hline Sample & & & & & \\
Loading $\left(\mu \mathrm{g} \mathrm{mg} \mathrm{mg}^{-1}\right)$ & $\begin{array}{l}\text { Entrapment efficiency } \\
(\%)\end{array}$ \\
\hline Blank PLGA NPs & $218.3 \pm 5.5$ & $-1.43 \pm 0.03$ & $-0.05 \pm 0.02$ & - & - \\
IR780@PLGA NPs & $260.6 \pm 4.8$ & $-2.35 \pm 0.02$ & $-0.17 \pm 0.06$ & $6.6 \pm 1.8$ & $31.2 \pm 1.2$
\end{tabular}


$\mathbf{A}_{12}$
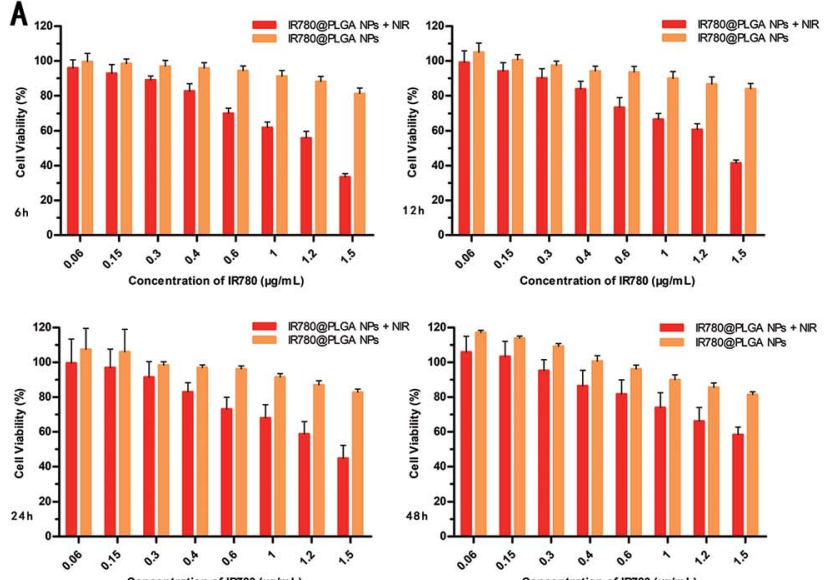

B

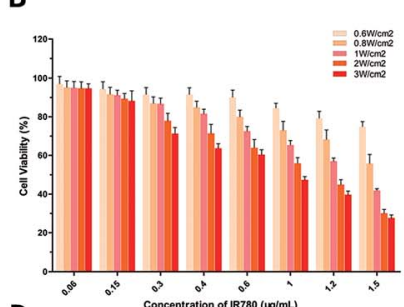

D

$0.06 \mu \mathrm{g} / \mathrm{mL}$

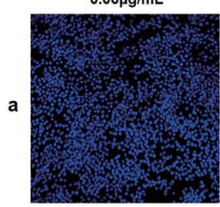

$0.3 \mu \mathrm{g} / \mathrm{mL}$
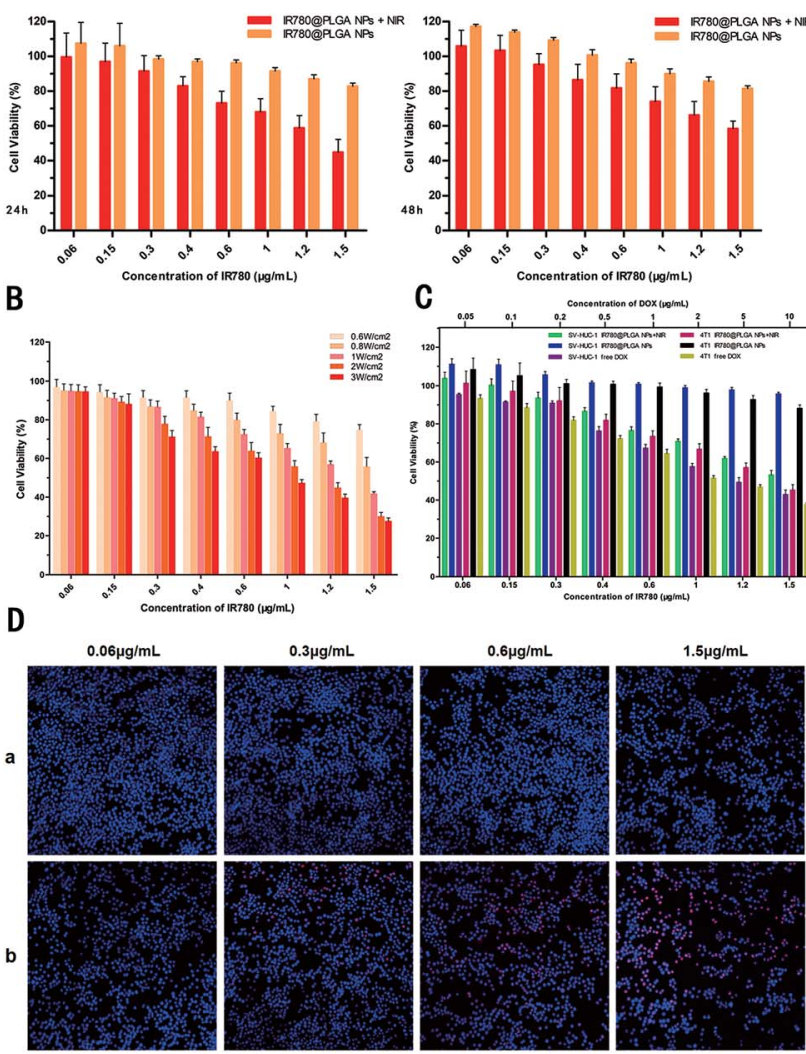

C
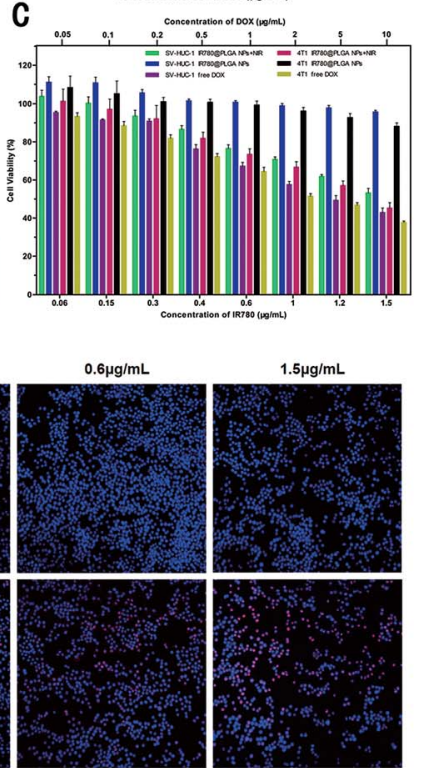

Fig. 4 The viability of 4T1 cells incubated with different concentrations of IR780 (A) with and without laser irradiation $\left(2.0 \mathrm{~W} \mathrm{~cm}^{-2}\right)(n=5)$ after $6,12,24$ and $48 \mathrm{~h}$. (B) Under different laser intensities $(0.6,0.8,1,2$ and $3 \mathrm{~W} \mathrm{~cm}^{-2}$ ). (C) Viability of SV-HUC-1 and 4T1 cells incubated with IR780@PLGA NPs and DOX. (D) Cytotoxicity evaluated by fluorescence microscopy using DAPI/PI double staining after different treatments: incubated with different concentrations of IR780@APLGA NPs (a) without NIR irradiation, (b) with NIR irradiation. Dead cells: red fluorescence of $\mathrm{Pl}$; nucleus: blue fluorescence of DAPI.

IR780@PLGA NPs under NIR irradiation $\left(2.0 \mathrm{~W} \mathrm{~cm}^{-2}, 10 \mathrm{~min}\right)$, the cell viability decreased because of the photothermal effect of IR780. The cell viability showed a marked decrease with increasing concentration of the IR780@PLGA NPs. Effective cellular growth inhibition of the IR780@PLGA NPs after NIR irradiation indicated that NIR-induced PTT enhanced the cancer cell killing effect $(p<0.05)$. The dead cell viability assay was further employed to visualize the cytotoxic effects of hyperthermia-assisted chemotherapy against 4T1 cells. The fluorescence in DAPI/PI iodide double staining was applied to identify dead/apoptotic cells after different treatments. As shown in Fig. 4D, when 4T1 cells were incubated with different concentrations of IR780@PLGA NPs without NIR laser exposure, almost no red fluorescence was observed, indicating negligible lethal effects. When $4 \mathrm{~T} 1$ cells were incubated with IR780@PLGA NPs under NIR irradiation for $10 \mathrm{~min}$, cell death was observed, demonstrating the IR780-triggered photothermal cytotoxicity. However, as shown in Fig. 4A, as the incubation time increased, the cell viability also increased. After incubation for more than $48 \mathrm{~h}$, the cell viability increased to greater than $50 \%$, indicating that the cells regrew rapidly enough for the therapy to fail. Moreover, as shown in Fig. 4B, after irradiation under different intensities of NIR light, the cell viability markedly decreased with increasing NIR light intensity. Interestingly, when incubated with IR780@PLGA NPs, SV-HUC-1 cells remained excellent vitality, indicating that normal human cells may have stronger resistance to IR780 (Fig. 4C). However, when irradiated with a NIR laser, the SV-HUC-1 cell viability decreased, showing that the IR780-triggered photothermal cytotoxicity could not be ignored even though normal human cells possess stronger resistance than cancer cells. It is also important that compared to DOX in a conventional therapeutic dose, ${ }^{5,38,40}$ IR780@NPs showed lower cytotoxicity; this suggests it to be an ideal material in cancer therapy with potential for clinical therapy. Notably, the IR780@PLGA NPs combined with PTT achieved exceptional cytotoxicity. These results indicate that the IR780@PLGA NPs effectively induce cell death through PTT therapy upon NIR laser irradiation.

\section{In vivo anti-tumour therapy}

To investigate the anti-tumour therapy in vivo, mice with 4T1 tumours in their legs were intra-tumourally injected with different solutions every other day for 8 days after first observing the bone damage caused by the tumour on the leg using ultrasound (Fig. 5). At 5 min post-injection, the tumours of the mice in each group were irradiated with the $808 \mathrm{~nm}$ laser $(2.0 \mathrm{~W}$ $\left.\mathrm{cm}^{-2}, 5 \mathrm{~min}\right)$. The temperature change during the irradiation of tumour tissue was measured, as shown in Fig. 6. The temperature of the tumour-bearing legs of the mice receiving the intratumoural injection of the IR780@PLGA NPs rapidly increased to $49.4^{\circ} \mathrm{C}$, which was greater than the temperature observed in the blank PLGA $\left(37.4^{\circ} \mathrm{C}\right)$ and PBS $\left(34.7^{\circ} \mathrm{C}\right)$ groups (Fig. $\left.6 \mathrm{~B}\right)$. The tumour volume and body weight were measured, as shown in Fig. 7C and D. In the PBS-injected group, the tumour volume markedly increased on day 8 compared with day 0. Compared with the original tumour volume on day 0 , the tumour volumes on day 8 increased by $197.1 \%$ for the PBS-injected group, $108.3 \%$ for the IR780@PLGA NP-injected group with NIR
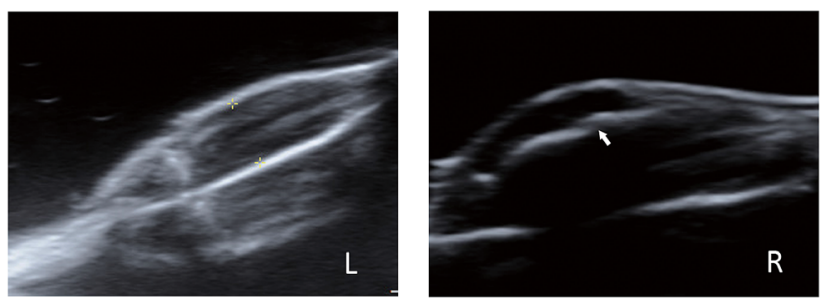

Fig. 5 Ultrasound images of a normal leg (L) and tumour-bearing leg (R). The arrow shows bone damage. 

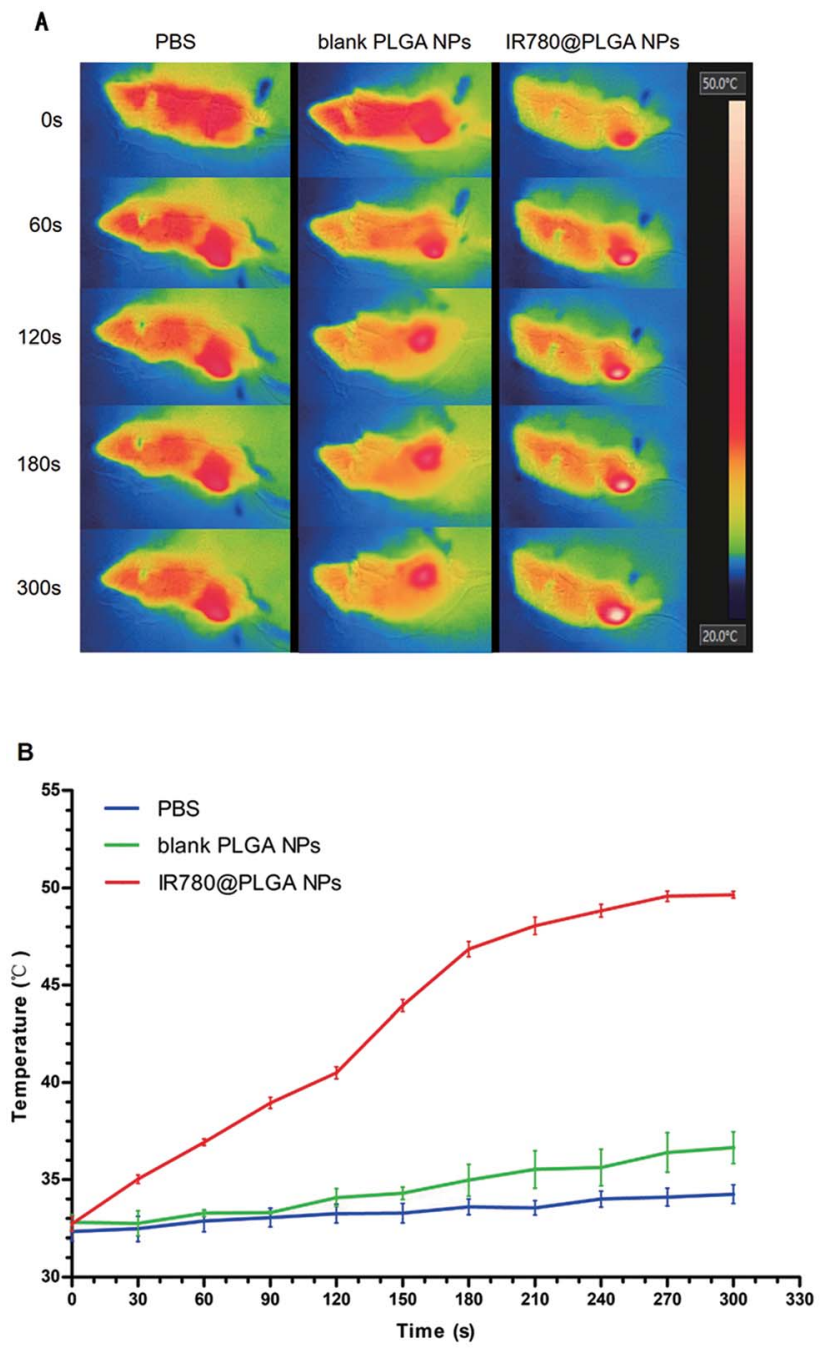

Fig. 6 In vivo PTT. Infrared thermal images of mice after irradiation (A) injected with PBS, blank PLGA NPs and IR780@PLGA NPs. (B) Temperature increases as a result of laser irradiation.

irradiation, and $151.1 \%, 152.2 \%$ and $176.8 \%$ for the IR780@PLGA NP group without NIR irradiation, blank PLGA NP group with NIR irradiation and blank PLGA NP group, respectively. These results suggest that IR780 has an obvious antitumour effect in vivo. For the blank PLGA NP group with NIR irradiation, the tumour volume slowly increased to $152.2 \%$ of the original tumour volume. Moreover, the tumour volume remained at nearly the same volume as the original tumour volume after the IR780@PLGA NPs were injected and exposed under laser irradiation. These results indicate that the combined therapy effectively inhibits tumour growth, and the combined anti-tumour effect was better than the anti-tumour effect of the individual components.

On days 0,4 , and 8 post-injection, 5 mice from each group were randomly selected, and the tumour-bearing legs were excised for micro CT analysis. Histopathological examination of the stained tissues was then conducted. Moreover, mice from each group were randomly selected on day 8 post-injection, and their organs were excised for histopathological examination.

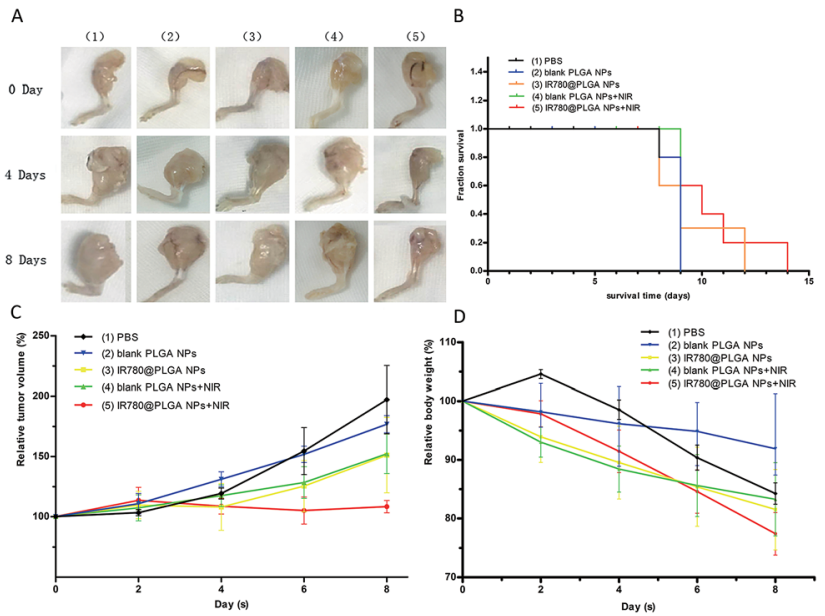

Fig. 7 In vivo anti-tumour therapy. (A) Images of tumour-bearing legs after administration of different treatments: (1) PBS, (2) blank PLGA NPs, (3) IR780 @PLGA NPs, (4) blank PLGA NPs + NIR (808 nm), and (5) IR780@PLGA NPs + NIR (808 nm). (B) Survival times, (C) tumour growth curves and (D) body weight curves of the mice after administration of different treatments: (1) PBS, (2) blank PLGA NPS, (3) IR780@PLGA NPs, (4) blank PLGA NPs + NIR (808 nm), and (5) IR780@PLGA NPs + NIR (808 nm). The tumour volumes were normalized to the corresponding initial tumour volumes, and the body weights were normalized to the corresponding initial body weights.

H\&E staining of the tumour-bearing legs treated with the IR780@PLGA NPs and NIR laser irradiation showed less bone destruction and more normal tissues around the bone than the mice in the other groups, indicating that a better inhibition effect was induced by this treatment (Fig. 8). Micro CT analysis indicated that the mice treated with the IR780@PLGA NPs and NIR laser irradiation exhibited minimal bone destruction (Fig. 10). These results demonstrate that further tumour cell growth was efficiently inhibited, and more effective cell apoptosis was observed as a result of the PTT therapy compared with the other treatments. In addition, H\&E staining analysis of

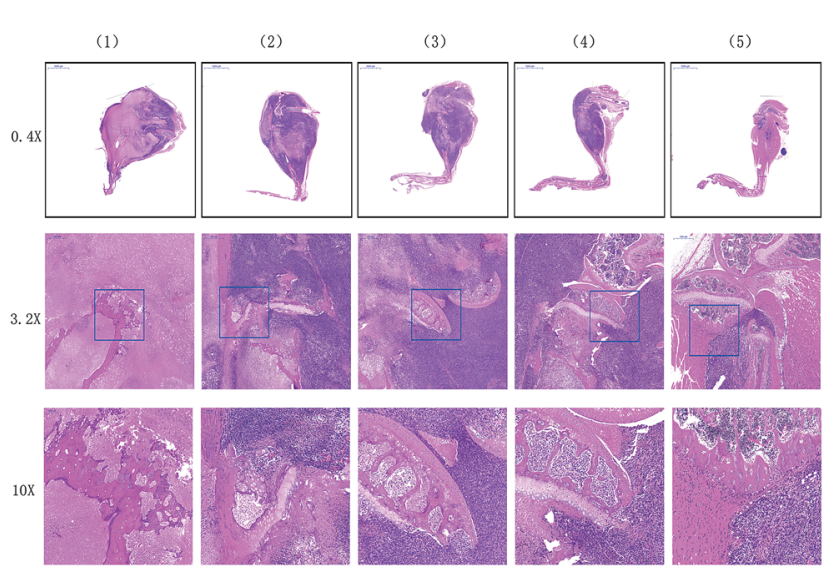

Fig. 8 H\&E staining of tumour tissue slices after administration of different treatments: (1) PBS, (2) blank PLGA NPs, (3) IR780@PLGA NPs, (4) blank PLGA NPs + NIR (808 nm), and (5) IR780@PLGA NPs + NIR (808 nm). 


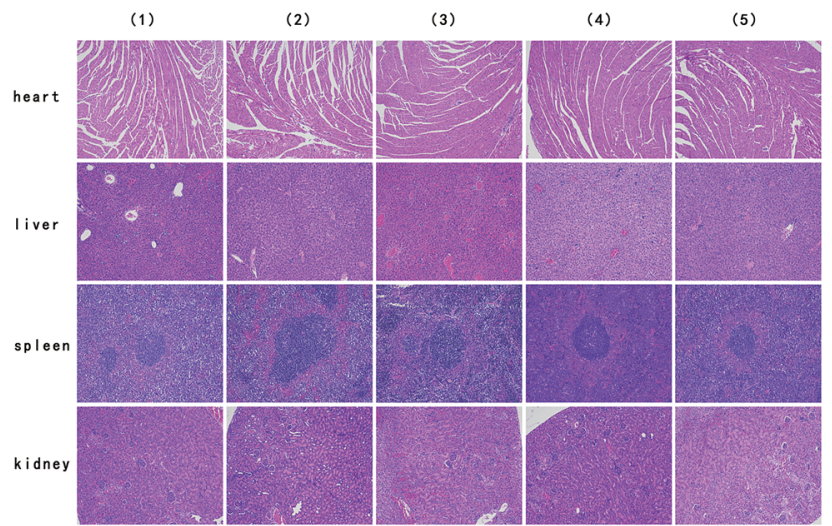

Fig. 9 H\&E staining images of different organ slices from the mice on day 8 after administration of different treatments: (1) PBS, (2) blank PLGA NPs, (3) IR780@PLGA NPs, (4) blank PLGA NPs + NIR (808 nm), and (5) IR780@PLGA NPs + NIR (808 nm).

the important organs indicated no obvious damage (Fig. 9). Thus, it was demonstrated that PTT using the IR780@PLGA NPs effectively suppresses the metastasis of breast cancer cells to bone and does not cause damage to normal organs.

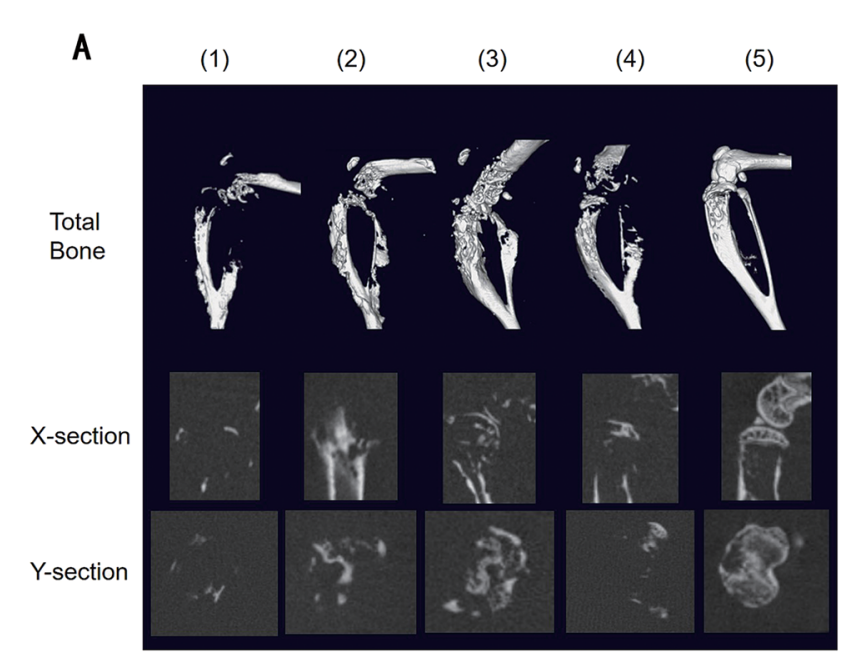

B

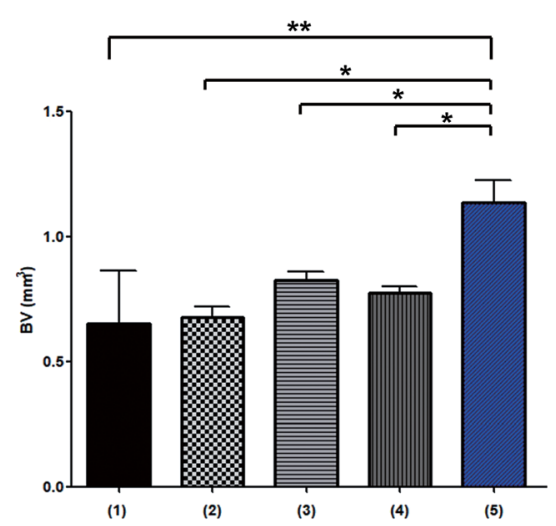

Fig. 10 Micro CT images (A) and data (B) for the following groups: (1) PBS, (2) blank PLGA NPs, (3) IR780@ PLGA NPs, (4) blank PLGA NPs + NIR $(808 \mathrm{~nm})$, and (5) IR780@PLGA NPs + NIR $(808 \mathrm{~nm})$. The data for each group are representative of at least three independent assays. ${ }^{*} p<0.05$.
Based on the results discussed above, the IR780@PLGA NPs and NIR laser irradiation showed outstanding PTT efficacy, which inhibited the metastasis of breast cancer cells to bone. We speculate that the excellent anti-tumour effects of the IR780@PLGA NPs are due to the following factors: (1) intratumoural injection of the IR780@PLGA NPs caused increased accumulation of the IR780@PLGA NPs in tumours, avoiding IR780 biodegradation in circulation in vivo; and (2) the effects of PTT effectively inhibited breast cancer proliferation and metastasis. Compared to traditional therapies such as surgery and chemotherapy, the IR780@PLGA NPs combined with PTT show pleasing therapeutic effects in inhibiting bone damage and possess lower cytotoxicity. Therefore, all of these advantages contribute to the excellent PTT effect of the IR780@PLGA NPs.

\section{Conclusions}

In this study, photothermally triggered IR780 nanomaterials based on PLGA NPs were fabricated to treat metastasized breast cancer cells in bone. The IR780@PLGA NPs showed excellent photothermal conversion properties in vitro and in vivo, and tumour growth was markedly inhibited by PTT. Treatment with the IR780@PLGA NPs combined with NIR laser irradiation eliminated the $4 \mathrm{~T} 1$ breast cancer cells in bone. Finally, the IR780@PLGA NPs show great potential for the treatment of bone metastases.

\section{Conflicts of interest}

There are no conflicts to declare. Animal experiments were performed in accordance with the guidelines of the Department of Laboratory Animals, Central South University, China, and were approved by the Ethics Committee of Central South University.

\section{Acknowledgements}

This work was supported by grants from the National Natural Science Foundation of China (Grant No. 81601883, 81873988), the Hunan Provincial Natural Science Foundation of China (Grant No. 2018JJ3861) and the Hunan Provincial Science \& Technology Department (Grant No. 2017SK50106).

\section{References}

1 F. Bray, J. Ferlay, I. Soerjomataram, R. L. Siegel, L. A. Torre and A. Jemal, Ca-Cancer J. Clin., 2018, 68, 394-424.

2 Z. Lin, Y. Liu, X. Ma, S. Hu, J. Zhang, Q. Wu, W. Ye, S. Zhu, D. Yang, D. Qu and J. Jiang, Sci. Rep., 2015, 5, 11709.

3 G. R. Mundy, Nat. Rev. Cancer, 2002, 2, 584-593.

4 R. Tanaka, K. Yonemori, A. Hirakawa, F. Kinoshita, N. Takahashi, J. Hashimoto, M. Kodaira, H. Yamamoto, M. Yunokawa, C. Shimizu, M. Fujimoto, Y. Fujiwara and K. Tamura, Oncologist, 2016, 21, 508-513.

5 Z. Yang, R. Cheng, C. Zhao, N. Sun, H. Luo, Y. Chen, Z. Liu, X. Li, J. Liu and Z. Tian, Theranostics, 2018, 8, 4097-4115. 
6 M. Cicek and M. J. Oursler, Cancer Metastasis Rev., 2006, 25, 635-644.

7 L. Zou, H. Wang, B. He, L. Zeng, T. Tan, H. Cao, X. He, Z. Zhang, S. Guo and Y. Li, Theranostics, 2016, 6, 762-772.

8 H. M. Chen, F. P. Chen, K. C. Yang and S. S. Yuan, JAMA Netw. Open, 2019, 2, e190429.

9 A. Yazdani, S. Dorri, A. Atashi, H. Shirafkan and H. Zabolinezhad, Breast Cancer, 2019, 13, 1178223419830978.

10 L. Wang and J. Yan, J. Photochem. Photobiol., B, 2019, 192, 68-73.

11 L. Wang, S. Chen, Y. Zhu, M. Zhang, S. Tang, J. Li, W. Pei, B. Huang and C. Niu, ACS Appl. Mater. Interfaces, 20180, 10, 42102-42114.

12 J. Sheng, L. Wang, Y. Han, W. Chen, H. Liu, M. Zhang, L. Deng and Y. N. Liu, Small, 2018, 14, 1702529.

13 R. E. Coleman, Cancer Treat. Rev., 2001, 27, 165-176.

14 Y. Yang, X. Yang, H. Li, C. Li, H. Ding, M. Zhang, Y. Guo and M. Sun, Colloids Surf., B, 2019, 173, 564-570.

15 K. N. Weilbaecher, T. A. Guise and L. K. McCauley, Nat. Rev. Cancer, 2011, 11, 411-425.

16 C. L. Chaffer and R. A. Weinberg, Science, 2011, 331, 15591564.

17 M. B. Meads, L. A. Hazlehurst and W. S. Dalton, Clin. Cancer Res., 2008, 14, 2519-2526.

18 X. Yang, H. Li, C. Qian, Y. Guo, C. Li, F. Gao, Y. Yang, K. Wang, D. Oupicky and M. Sun, Nanomedicine, 2018, 14, 2283-2294.

19 X. Qiu, L. Xu, Y. Zhang, A. Yuan, K. Wang, X. Zhao, J. Wu, H. Guo and Y. Hu, Mol. Pharm., 2016, 13, 829-838.

20 Q. Chen, C. Liang, X. Wang, J. He, Y. Li and Z. Liu, Biomaterials, 2014, 35, 9355-9362.

21 C. Wang, L. Xu, C. Liang, J. Xiang, R. Peng and Z. Liu, Adv. Mater., 2014, 26, 8154-8162.

22 C. Niu, Y. Xu, S. An, M. Zhang, Y. Hu, L. Wang and Q. Peng, Sci. Rep., 2017, 7, 5490.

23 F. Guo, M. Yu, J. Wang, F. Tan and N. Li, ACS Appl. Mater. Interfaces, 2015, 7, 20556-20567.
24 H. Li, X. Yang, Z. Zhou, K. Wang, C. Li, H. Qiao, D. Oupicky and M. Sun, J. Controlled Release, 2017, 261, 126-137.

25 H. Li, K. Wang, X. Yang, Y. Zhou, Q. Ping, D. Oupicky and M. Sun, Acta Biomater., 2017, 53, 399-413.

26 C. Jiang, H. Cheng, A. Yuan, X. Tang, J. Wu and Y. Hu, Acta Biomater., 2015, 14, 61-69.

27 C. L. Peng, Y. H. Shih, P. C. Lee, T. M. Hsieh, T. Y. Luo and M. J. Shieh, ACS Nano, 2011, 5, 5594-5607.

28 S. Li, J. Johnson, A. Peck and Q. Xie, J. Transl. Med., 2017, 15, 18.

29 S. Li, S. Zhou, Y. Li, X. Li, J. Zhu, L. Fan and S. Yang, ACS Appl. Mater. Interfaces, 2017, 9, 22332-22341.

30 K. Wang, Y. Zhang, J. Wang, A. Yuan, M. Sun, J. Wu and Y. Hu, Sci. Rep., 2016, 6, 27421.

31 C. Yue, P. Liu, M. Zheng, P. Zhao, Y. Wang, Y. Ma and L. Cai, Biomaterials, 2013, 34, 6853-6861.

32 W. Li, J. Peng, Q. Yang, L. Chen, L. Zhang, X. Chen and Z. Qian, Biomater. Sci., 2018, 6, 1201-1216.

33 C. Pais-Silva, D. de Melo-Diogo and I. J. Correia, Eur. J. Pharm. Biopharm., 2017, 113, 108-117.

34 Q. Chen, C. Liang, C. Wang and Z. Liu, Adv. Mater., 2015, 27, 903-910.

35 C. G. Alves, R. Lima-Sousa, D. de Melo-Diogo, R. O. Louro and I. J. Correia, Int. J. Pharm., 2018, 542, 164-175.

36 C. K. Ramanlal, A. Kumar, K. V. Megraj, M. Ukawala, A. S. Manjappa, A. K. Mishra, J. Monkkonen and M. R. Ramachandra, J. Controlled Release, 2012, 158, 470478.

37 D. M. Valcourt, M. N. Dang and E. S. Day, J. Biomed. Mater. Res., 2019, 107, 1702-1712.

38 Y. Deng, F. Kafer, T. Chen, Q. Jin, J. Ji and S. Agarwal, Small, 2018, 14, e1802420.

39 B. Hildebrandt, P. Wust, O. Ahlers, A. Dieing, G. Sreenivasa, T. Kerner, R. Felix and H. Riess, Crit. Rev. Oncol.-Hematol., 2002, 43, 33-56.

40 J. Xu, D. Liu, H. Niu, G. Zhu, Y. Xu, D. Ye, J. Li and Q. Zhang, J. Exp. Clin. Cancer Res., 2017, 36, 19. 